\title{
Editorial: Degradation of Cultural Heritage Artifacts: From Archaeometry to Materials Development
}

\author{
Radu Claudiu Fierascu ${ }^{1,2 *}$, Enrico Sassoni ${ }^{3}$, Alina Catrinel Ion $^{2}$, Anton Ficai $^{2}$ and \\ Elwira Sieniawska ${ }^{4}$ \\ ${ }^{1}$ National Institute for Research \& Development in Chemistry and Petrochemistry-ICECHIM, Bucharest, Romania, ${ }^{2}$ Politehnica \\ University of Bucharest, Bucharest, Romania, ${ }^{3}$ Department of Civil, Chemical, Environmental, and Materials Engineering, \\ University of Bologna, Bologna, Italy, ${ }^{4}$ Department of Pharmacognosy, Medical University of Lublin, Lublin, Poland
}

Keywords: degradation, archaeometry, artifacts, restoration, conservation, natural materials, synthetic materials

\section{Editorial on the Research Topic}

\section{Degradation of Cultural Heritage Artifacts: From Archaeometry to Materials Development}

Cultural heritage studies not only represent an important research area for scientists in various areas of materials science, but the results obtained also provide the basis for choosing the appropriate restoration/conservation strategy. The degradation of cultural heritage materials represents one of

\section{OPEN ACCESS}

Edited and reviewed by: V. S. Raja, Indian Institute of Technology Bombay, India

*Correspondence: Radu Claudiu Fierascu fierascu.radu@icechim.ro

Specialty section: This article was submitted to Environmental Degradation of Materials,

a section of the journal

Frontiers in Materials

Received: 02 June 2021 Accepted: 22 June 2021 Published: 02 July 2021

Citation:

Fierascu RC, Sassoni E, Ion AC, Ficai $A$ and Sieniawska E (2021) Editorial: Degradation of Cultural Heritage Artifacts: From Archaeometry to Materials Development. Front. Mater. 8:719685. doi: 10.3389/fmats.2021.719685 the most important threats for their preservation. The study of cultural heritage artifacts aims at identifying, besides the nature of the materials, their state of degradation and the degradation products affecting them. The restoration procedure should start with the study of the artifacts support material, followed by the development/identification of the materials to be used for intervention. The proposal of materials for cultural heritage preservation should be supported by studies covering their long-term effect on the artifacts.

Although many advances were recorded in this area in the last decades, the search for alternative procedures and materials for the restoration/conservation of cultural heritage artifacts is still in full progress, because many important issues have not been solved yet and/or new threats have arisen with time (e.g., climate change). The Research Topic was intended to bring together specialists with different backgrounds (e.g., materials science, natural products, analytic chemistry, restoration/ conservation), in order to offer the readers a comprehensive image of recent developments and perspectives in the area of cultural heritage artifacts degradation pathways and new materials for preventing it. The collection gathered seven excellent original research, covering several aspects of cultural heritage study.

Ghervase et al. investigated five bronze adornment objects from the First Iron Age (ninth-eighth c. BC), discovered at Tărtăria-Podu Tărtăriei vest archaeological site. The applied methods included digital radiography (to assess the physical state of the objects and to identify potential specific craftsmanship details), X-ray fluorescence (XRF) and laser-induced breakdown spectroscopy (LIBS) to identify the materials and to stratigraphically evaluate the objects. The results revealed a good preservation state of the objects, as well as a high variability of all elements, accounted by a combination of factors, including the inhomogeneous nature of the raw material, and migration of elements.

Cortea et al. applied a multi-analytic approach for the investigation of a degraded Jewish ritual parchment. The selected noninvasive investigations included multi- and hyperspectral imaging, Fourier transform infrared spectroscopy (FTIR) and XRF. The results allowed the mapping of 
previous conservation interventions, forms of degradation, and the presence of two different types of inks (iron gall, respectively carbon-based ink). The authors also proposed an algorithm for the reconstruction of degraded letters. XRF and FTIR analyses provided information on the chemical fingerprint of inks, denaturation of the collagen fibers, the presence of fungalderived calcium oxalates and zinc carboxylates, as well as the use of different compounds in the parchment manufacture process (calcium sulfate, vegetable tannins, oils, other elements).

Fierascu et al. proposed an analytical methodology to characterize seven ancient ceramic objects (dating to the Late Bronze Age), involving the use of optical microscopy (revealing strong inhomogeneities on the surface), XRF, X-ray diffraction (XRD), FTIR, and thermal analysis. The results obtained by XRF and XRD offered information regarding mineralogical composition of the samples (some samples presenting illite/ muscovite and plagioclase phases in higher quantities or a lower quartz content), also confirmed by FTIR analysis. The thermal analysis completes the analytical investigation of the ceramic samples. The thermal behavior of the sample conducted to some explanation regarding raw materials (that the major clay mineral in the samples is represented by illite) or to environmental factors during their burial in the soil.

Loureiro et al. quantified the components of historic mortars from Belém do Pará (Brazil), by wet chemical analysis, XRD, differential scanning calorimetry (DSC) and XRF, in order to determine the possibility of estimating the binder/aggregate ratio. The results showed that the amounts of the components in the mortars could be quantified with the use of approximately $3 \mathrm{~g}$ of sample, leading to the development of appropriate restoration mortar. The authors suggest, however, whenever possible, a combination of techniques for the estimation, necessary to obtain a more reliable and complete characterization.

The photodegradation kinetics of alkyd paints was studied by Pagnin et al. The authors presented the degradation behavior of artificial ultramarine blue, hydrated chromium oxide green, and cadmium sulfate yellow pigments mixed with alkyd resin and aged under simulated sunlight exposure for a total of $1,008 \mathrm{~h}$. The samples were also characterized by $3 \mathrm{D}$ optical microscopy, scanning electron microscopy, colorimetry analyses, Attenuated Total Reflection Infrared Spectroscopy, also carrying out multivariate analysis of microscopic images, in order to study the degradation effects linked to color and texture changes. The conclusion of the study was that the degradation processes of alkyd resin are influenced by the presence of the different inorganic pigments used and their concentration in the mixtures.
Edith Joseph proposed a biopassivation method (based on the use of selected microorganisms) for preserving copper-based artefacts. The method is based on the capacity of some fungi to form copper oxalates on the corroded surface of copper alloys. The behavior and performance of the proposed treatment was evaluated during natural aging procedures (suggesting that the method stabilizes the corrosion process, with lesser chromatic variation, compared to traditional methods) and was validated on real cases (outdoor sculptures and archaeological objects), the work being finalized with the proposal of a ready-to-use kit.

Finally, Molina et al. determined the influence of the roughness of surface finish on the durability of different types of natural stone (calcarenites, travertine, and sandstone), and, in the same time, evaluated the efficiency of a calcium phosphatebased consolidant against deterioration in polluted urban environments, by exposing treated samples to artificial atmospheres with $\mathrm{SO}_{2}$ pollution. The conclusions of the authors revealed that all the surface finishes were vulnerable to the decay in acidic atmospheres (the saw-cut finish being the least affected). Also, correlated with their mineral composition and texture, the travertine and sandstone were more resistant to deterioration than the calcarenites. The applied consolidant improved the behavior of the samples, making them more resistant to acid attack without significantly altering the water vapor permeability, the color or the roughness of the surface.

\section{AUTHOR CONTRIBUTIONS}

All authors listed have made a substantial, direct and intellectual contribution to the work, and approved it for publication.

\section{ACKNOWLEDGMENTS}

We thank all the authors, reviewers, and editorial board members for their great contributions to this Research Topic.

Conflict of Interest: The authors declare that the research was conducted in the absence of any commercial or financial relationships that could be construed as a potential conflict of interest.

Copyright (c) 2021 Fierascu, Sassoni, Ion, Ficai and Sieniawska. This is an openaccess article distributed under the terms of the Creative Commons Attribution License (CC BY). The use, distribution or reproduction in other forums is permitted, provided the original author(s) and the copyright owner(s) are credited and that the original publication in this journal is cited, in accordance with accepted academic practice. No use, distribution or reproduction is permitted which does not comply with these terms. 